\title{
Nonstationary Stimulated Raman Scattering by Polaritons in Continuum of Dipole-Active Phonons
}

\author{
Vladimir Feshchenko1, Galina Feshchenko² \\ ${ }^{1}$ Dawson College, Montreal, Canada \\ ${ }^{2}$ Vanier College, Montreal, Canada \\ Email:vfeshchenko@dawsoncollege.qc.ca, feshcheg@vaniercollege.qc.ca
}

How to cite this paper: Feshchenko, V. and Feshchenko, G. (2018) Nonstationary Stimulated Raman Scattering by Polaritons in Continuum of Dipole-Active Phonons. Journal of Applied Mathematics and Physics, 6, 405-417.

https://doi.org/10.4236/jamp.2018.62038

Received: August 12, 2017

Accepted: February 24, 2018

Published: February 27, 2018

Copyright $\odot 2018$ by authors and Scientific Research Publishing Inc. This work is licensed under the Creative Commons Attribution International License (CC BY 4.0).

http://creativecommons.org/licenses/by/4.0/

\begin{abstract}
The system of equations simulating the processes of nonstationary stimulated Raman scattering (SRS) with the excitation of polar optical phonons is obtained. This system is found by applying such standard methods as the nonstationary theory of perturbations, which resulted in the equations for the amplitudes of probabilities to find the discrete system in certain state, and slowly-varying amplitudes for the electromagnetic waves. It has been shown that the obtained system includes, as extremes, the case of classical interaction between electromagnetic field and resonant medium (including the "area theorem"), and the one related with SRS on optical phonons. We have conducted both theoretical and numerical investigation of simplified system assuming that the amplitudes of all electromagnetic waves (laser, Stokes, and polariton) were real (there was no destructive spatial-temporal phase modulation). Only low-order nonlinear processes are considered. It is shown that this system can be reduced to Sine-Gordon equation. This system can also be simplified to the equation that simulates the motion of physical pendulum from upper equilibrium position. The numerical study of nonstationary SRS when the electromagnetic field of laser radiation and Stokes excite both polariton emission and the continuum of dipole-active phonons has been carried out. The evolution of the intensity of the polariton wave as function of the length of nonlinear medium has been numerically analyzed.
\end{abstract}

\section{Keywords}

Nonstationary Stimulated Raman Scattering, Polaritons

\section{Introduction}

Over the past two decades, the field of polaritons (exciton-like, plasmon-like, 
and phonon-like) has been substantially developed [1] [2]. For instance, the theory of exciton-polaritons provides the successful explanation of physical phenomena in microcavities, which is essential for the design of polariton lasers [3] [4]. The spectroscopy of complex semiconductor layer structures is related with the theory of plasmon-polaritons [5] [6]. The progress in infrared spectroscopy of ionic crystals is based on theory of phonon-polaritons [7] [8] [9]. Surface polaritons are considered in details in [10]. However, given the complexity of the equations simulating multi-photon processes in nonlinear media and accepted simplifications (the method of slowly-varying amplitudes, the given field approximation, phase capture, etc.) some results still remain unforeseen [11] [12] [13] [14]. In this paper, we made an attempt to obtain and solve the system of equations that would properly describe the processes of nonstationary SRS in ionic crystals by phonon-polaritons. The Section 2 of our paper is based on technique developed in [15] where the equations for the "nonresonant" amplitude of probabilities to find the particle at certain level were approximate whereas the equations for the "resonant" amplitudes of probabilities $a_{1}$ and $a_{2}$ were exact. Despite the fact that the obtained system was cumbersome, we managed to bring it to the compact form. In Section 3, we have compared the theoretical and experimental gain factors [16]-[25]. In Section 4, we found the simplified system of equations, showed that this system corresponds to the extreme cases studied previously (see [26] [27]), and reduced the system to Sine-Gordon equation [28]. The numerical analysis of the system has been carried out as well.

\section{Basic Principles and Equations}

In this paper we assume that two optical pulses with frequencies $\omega_{1}$ (the frequency of laser pump) and $\omega_{2}$ (that of Stokes) propagate in crystal at the angles $\tilde{\theta}_{1,2}$ with respect to $\mathrm{z}$-axis, which is perpendicular to the input surface of the medium. The laser wave $\omega_{1}$ and Stokes $\omega_{2}$ during SRS generate $\omega_{3}=\omega_{1}-\omega_{2}$ as well as the polar optical phonon $\omega_{f} \equiv \omega_{21}$ in the vicinity of which falls $\omega_{3} \quad\left(\omega_{3}=\omega_{21}+\Delta \omega\right)$.

We apply the standard equations for the amplitude of probability $a_{k}$ of finding the system in state with energy $E_{k}$ [29] [30]:

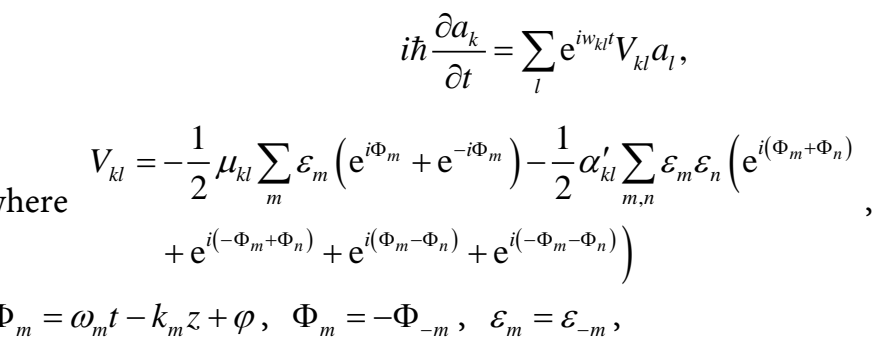

$\mu_{k l}$ is the dipole moment of the transition $k \rightarrow l ; \omega_{m}, k_{m}, \varepsilon_{m}$ and $\varphi_{m}$ are the frequencies, $\mathrm{z}$-components of wave vectors, real "slowly-varying amplitudes", and phases of the interacting waves, accordingly; $\alpha_{k l}^{\prime}$ is the tensor of the combinational scattering.

Then we use (1) to find $a_{l} \quad(l \neq 1,2)$. 


$$
\begin{aligned}
a_{l}= & \frac{1}{2 \hbar} \sum_{m} \mu_{l 1} \varepsilon_{m} \mathrm{e}^{i \omega_{l 1} t}\left(\frac{\mathrm{e}^{i \Phi_{m}}}{\omega_{l 1}+\omega_{m}}+\frac{\mathrm{e}^{-i \Phi_{m}}}{\omega_{l 1}-\omega_{m}}\right) a_{1}+\frac{1}{2 \hbar} \sum_{m} \mu_{l 2} \varepsilon_{m} \mathrm{e}^{i \omega_{l 2} t}\left(\frac{\mathrm{e}^{i \Phi_{m}}}{\omega_{l 2}+\omega_{m}}\right. \\
& \left.+\frac{\mathrm{e}^{-i \Phi_{m}}}{\omega_{l 2}-\omega_{m}}\right) a_{2}+\frac{1}{2 \hbar} \sum_{m, n} \alpha_{l 1}^{\prime} \varepsilon_{m} \varepsilon_{n} \mathrm{e}^{i \omega_{l 1} t}\left(\frac{\mathrm{e}^{i\left(\Phi_{m}+\Phi_{n}\right)}}{\omega_{l 1}+\omega_{m}+\omega_{n}}+\frac{\mathrm{e}^{i\left(-\Phi_{m}+\Phi_{n}\right)}}{\omega_{l 1}-\omega_{m}+\omega_{n}}\right. \\
& \left.+\frac{\mathrm{e}^{i\left(\Phi_{m}-\Phi_{n}\right)}}{\omega_{l 1}+\omega_{m}-\omega_{n}}+\frac{\mathrm{e}^{i\left(-\Phi_{m}-\Phi_{n}\right)}}{\omega_{l 1}-\omega_{m}-\omega_{n}}\right) a_{1}+\frac{1}{2 \hbar} \sum_{m, n} \alpha_{l 2}^{\prime} \varepsilon_{m} \varepsilon_{n} \mathrm{e}^{i \omega_{l 2} t}\left(\frac{\mathrm{e}^{i\left(\Phi_{m}+\Phi_{n}\right)}}{\omega_{l 2}+\omega_{m}+\omega_{n}}\right. \\
& \left.+\frac{\mathrm{e}^{i\left(-\Phi_{m}+\Phi_{n}\right)}}{\omega_{l 2}-\omega_{m}+\omega_{n}}+\frac{\mathrm{e}^{i\left(\Phi_{m}-\Phi_{n}\right)}}{\omega_{l 2}+\omega_{m}-\omega_{n}}+\frac{\mathrm{e}^{i\left(-\Phi_{m}-\Phi_{n}\right)}}{\omega_{l 2}-\omega_{m}-\omega_{n}}\right) a_{2},(l \neq 1,2)
\end{aligned}
$$

To obtain the exact equations for $a_{1,2}$ we substitute (2) into (1):

$$
\begin{aligned}
& i \hbar \frac{\partial a_{1}}{\partial t}=-\left\{r_{1} \cdot \varepsilon_{1}^{2}+r_{2} \cdot \varepsilon_{2}^{2}+r_{3} \cdot \varepsilon_{3}^{2}+\left[r 1_{+}+r 2_{-}\right] \cdot \varepsilon_{1} \varepsilon_{2} \varepsilon_{3} \mathrm{e}^{-i \theta}+\left[r 1_{-}+r 2_{+}\right]\right. \\
& \cdot \varepsilon_{1} \varepsilon_{2} \varepsilon_{3} \mathrm{e}^{\mathrm{i} \theta}+r 3_{1} \cdot \varepsilon_{1}^{4}+r 3_{2} \cdot \varepsilon_{2}^{4}+r 3_{3} \cdot \varepsilon_{3}^{4}+r 4_{12} \cdot \varepsilon_{1}^{2} \varepsilon_{2}^{2}+r 4_{13} \cdot \varepsilon_{1}^{2} \varepsilon_{3}^{2} \\
& \left.+r 4_{23} \cdot \varepsilon_{2}^{2} \varepsilon_{3}^{2}\right\} a_{1}-\mathrm{e}^{i \Delta \omega t}\left\{\frac{1}{2} \mu_{12} \varepsilon_{3} \mathrm{e}^{i\left(-k_{3} z+\varphi_{3}\right)}+\left[\alpha_{12}^{\prime}+r 5\right] \varepsilon_{1} \varepsilon_{2} \mathrm{e}^{i\left(k_{2}-k_{1}\right) z}\right. \\
& \cdot \mathrm{e}^{i\left(\varphi_{1}-\varphi_{2}\right)}+\left[r 6_{31} \cdot \varepsilon_{1}^{2} \varepsilon_{3}+r 6_{32} \cdot \varepsilon_{2}^{2} \varepsilon_{3}+r 7 \cdot \varepsilon_{3}^{3}\right] \mathrm{e}^{i\left(-k_{3} z+\varphi_{3}\right)} \\
& +r 8_{12,+} \varepsilon_{1}^{3} \varepsilon_{2} \mathrm{e}^{i\left(k_{2}-k_{1}\right) z} \mathrm{e}^{i\left(\varphi_{1}-\varphi_{2}\right)}+r 8_{21,-} \cdot \varepsilon_{1} \varepsilon_{2}^{3} \mathrm{e}^{i\left(k_{2}-k_{1}\right) z} \mathrm{e}^{i\left(\varphi_{1}-\varphi_{2}\right)} \\
& \left.+r 9 \cdot \varepsilon_{1} \varepsilon_{2} \varepsilon_{3}^{2} \mathrm{e}^{i\left(k_{2}-k_{1}\right) z} \mathrm{e}^{i\left(\varphi_{1}-\varphi_{2}\right)}+r 10 \cdot \varepsilon_{1} \varepsilon_{2} \varepsilon_{3}^{2} \mathrm{e}^{i\left(k_{1}-k_{2}-2 k_{3}\right) z} \mathrm{e}^{i\left(\varphi_{2}+2 \varphi_{3}-\varphi_{1}\right)}\right\} a_{2} \\
& i \hbar \frac{\partial a_{2}}{\partial t}=-\mathrm{e}^{-i \Delta \omega t}\left\{\frac{1}{2} \mu_{21} \cdot \varepsilon_{3} \mathrm{e}^{i\left(k_{3} z-\phi_{3}\right)}+\left[\alpha_{21}^{\prime}+p 5\right] \cdot \varepsilon_{1} \varepsilon_{2} \mathrm{e}^{i\left(k_{1}-k_{2}\right) z} \mathrm{e}^{i\left(\varphi_{2}-\varphi_{1}\right)}\right. \\
& +\left[p 6_{31} \cdot \varepsilon_{1}^{2} \varepsilon_{3}+p 6_{32} \cdot \varepsilon_{2}^{2} \varepsilon_{3}+p 7 \cdot \varepsilon_{3}^{3}\right] \cdot \mathrm{e}^{i\left(k_{3} z-\varphi_{3}\right)}+p 8_{12,+} \cdot \varepsilon_{1}^{3} \varepsilon_{2} \mathrm{e}^{i\left(k_{1}-k_{2}\right) z} \\
& \cdot \mathrm{e}^{i\left(\varphi_{2}-\varphi_{1}\right)}+p 8_{12,-} \cdot \varepsilon_{1} \varepsilon_{2}^{3} \mathrm{e}^{i\left(k_{1}-k_{2}\right) z} \mathrm{e}^{i\left(\varphi_{2}-\varphi_{1}\right)}+p 9 \cdot \varepsilon_{1} \varepsilon_{2} \varepsilon_{3}^{2} \mathrm{e}^{i\left(k_{1}-k_{2}\right) z} \mathrm{e}^{i\left(\varphi_{2}-\varphi_{1}\right)} \\
& \left.+p 10 \cdot \varepsilon_{1} \varepsilon_{2} \varepsilon_{3}^{2} \mathrm{e}^{i\left(2 k_{3}+k_{2}-k_{1}\right) z} \mathrm{e}^{i\left(\varphi_{1}-\varphi_{2}-2 \varphi_{3}\right)}\right\} a_{1}-\left\{p_{1} \cdot \varepsilon_{1}^{2}+p_{2} \cdot \varepsilon_{2}^{2}+p_{3} \cdot \varepsilon_{3}^{2}\right. \\
& +\left[p 1_{+}+p 2_{-}\right] \varepsilon_{1} \varepsilon_{2} \varepsilon_{3} \mathrm{e}^{-i \theta}+\left[p 1_{-}+p 2_{+}\right] \varepsilon_{1} \varepsilon_{2} \varepsilon_{3} \mathrm{e}^{i \theta}+p 3_{1} \cdot \varepsilon_{1}^{4}+p 3_{2} \cdot \varepsilon_{2}^{4} \\
& \left.+p 3_{3} \cdot \varepsilon_{3}^{4}+p 4_{12} \cdot \varepsilon_{1}^{2} \varepsilon_{2}^{2}+p 4_{13} \cdot \varepsilon_{1}^{2} \varepsilon_{3}^{2}+p 4_{23} \cdot \varepsilon_{2}^{2} \varepsilon_{3}^{2}\right\} a_{2} \\
& \text { where } r_{i} \equiv \frac{1}{2 \hbar} \sum_{l \neq 1,2} \mu_{11} \mu_{l 1} \frac{\omega_{l 1}}{\omega_{l 1}^{2}-\omega_{i}^{2}} \text {; } \\
& r 1_{ \pm} \equiv \frac{1}{2 \hbar} \sum_{l \neq 1,2} \mu_{11} \alpha_{l 1}^{\prime}\left[\frac{1}{\omega_{l 1} \pm \omega_{3} \mp \omega_{1}}+\frac{1}{\omega_{l 1} \pm \omega_{2} \mp \omega_{1}}+\frac{1}{\omega_{l 1} \pm \omega_{3} \pm \omega_{2}}\right] \\
& r 2_{ \pm} \equiv \frac{1}{2 \hbar} \sum_{l \neq 1,2} \alpha_{11}^{\prime} \mu_{l 1}\left[\frac{1}{\omega_{l 1} \mp \omega_{3}}+\frac{1}{\omega_{l 1} \mp \omega_{2}}+\frac{1}{\omega_{l 1} \pm \omega_{1}}\right] \text {; } \\
& r 3_{i} \equiv \frac{1}{4 \hbar} \sum_{l \neq 1,2} \alpha_{11}^{\prime} \alpha_{l 1}^{\prime}\left[\frac{1}{\omega_{l 1}+2 \omega_{i}}+\frac{4}{\omega_{l 1}}+\frac{1}{\omega_{l 1}-2 \omega_{i}}\right] \text {; } \\
& r 4_{i j} \equiv \frac{1}{\hbar} \sum_{l \neq 1,2} \alpha_{11}^{\prime} \alpha_{l 1}^{\prime}\left[\frac{1}{\omega_{l 1}+\omega_{i}+\omega_{j}}+\frac{1}{\omega_{l 1}-\omega_{i}+\omega_{j}}\right. \\
& \left.+\frac{1}{\omega_{l 1}+\omega_{i}-\omega_{j}}+\frac{1}{\omega_{l 1}-\omega_{i}-\omega_{j}}+\frac{2}{\omega_{l 1}}\right] \text {; }
\end{aligned}
$$




$$
\begin{aligned}
& r 5 \equiv \frac{1}{4 \hbar} \sum_{l \neq 1,2} \mu_{11} \mu_{l 2}\left[\frac{1}{\omega_{l 2}+\omega_{1}}+\frac{1}{\omega_{l 2}-\omega_{2}}\right] \\
& r 6_{i j} \equiv \frac{1}{2 \hbar} \sum_{l \neq 1,2} \mu_{1 l} \alpha_{l 2}^{\prime}\left[\frac{1}{\omega_{l 2}+\omega_{i}-\omega_{j}}+\frac{1}{\omega_{l 2}+\omega_{i}+\omega_{j}}+\frac{1}{\omega_{l 2}}\right] \\
& +\frac{1}{2 \hbar} \sum_{l \neq 1,2} \alpha_{11}^{\prime} \mu_{l 2}\left[\frac{1}{\omega_{l 2}+\omega_{i}}+\frac{1}{\omega_{l 2}+\omega_{j}}+\frac{1}{\omega_{l 2}-\omega_{j}}\right] \text {; } \\
& r 7 \equiv \frac{1}{4 \hbar} \sum_{l \neq 1,2} \mu_{1 l} \alpha_{l 2}^{\prime}\left[\frac{1}{\omega_{l 2}+2 \omega_{3}}+\frac{2}{\omega_{l 2}}\right]+\frac{1}{4 \hbar} \sum_{l \neq 1,2} \alpha_{1 l}^{\prime} \mu_{l 2} \cdot\left[\frac{2}{\omega_{l 2}+\omega_{3}}+\frac{1}{\omega_{l 2}-\omega_{3}}\right] \\
& r 8_{i j, \pm} \equiv \frac{1}{2 \hbar} \sum_{l \neq 1,2} \alpha_{11}^{\prime} \alpha_{l 2}^{\prime}\left[\frac{1}{\omega_{l 2} \pm 2 \omega_{i}}+\frac{2}{\omega_{l 2}}+\frac{2}{\omega_{l 2}+\omega_{1}-\omega_{2}}+\frac{1}{\omega_{l 2} \mp \omega_{i} \mp \omega_{j}}\right] \text {; } \\
& r 9 \equiv \frac{1}{\hbar} \sum_{l \neq 1,2} \alpha_{11}^{\prime} \alpha_{l 2}^{\prime}\left[\frac{1}{\omega_{12}+\omega_{1}+\omega_{3}}+\frac{1}{\omega_{12}+\omega_{1}-\omega_{3}}+\frac{1}{\omega_{12}+\omega_{1}-\omega_{2}}\right. \\
& \left.+\frac{1}{\omega_{12}-\omega_{2}+\omega_{3}}+\frac{1}{\omega_{12}-\omega_{2}-\omega_{3}}+\frac{1}{\omega_{12}}\right] \\
& r 10 \equiv \frac{1}{2 \hbar} \sum_{l \neq 1,2} \alpha_{11}^{\prime} \alpha_{l 2}^{\prime}\left[\frac{2}{\omega_{l 2}-\omega_{1}+\omega_{3}}+\frac{1}{\omega_{l 2}-\omega_{1}+\omega_{2}}\right. \\
& \left.+\frac{2}{\omega_{l 2}+\omega_{2}+\omega_{3}}+\frac{1}{\omega_{l 2}-\omega_{1}+\omega_{3}}\right] \\
& \theta \equiv\left(k_{2}+k_{3}-k_{1}\right) z+\left(\varphi_{1}-\varphi_{2}-\varphi_{3}\right) ; \quad p_{i} \equiv \frac{1}{2 \hbar} \sum_{l \neq 1,2} \mu_{2 l} \mu_{l 2} \frac{\omega_{l 2}}{\omega_{l 2}^{2}-\omega_{i}^{2}} \\
& p 1_{ \pm} \equiv \frac{1}{2 \hbar} \sum_{l \neq 1,2} \mu_{2 l} \alpha_{l 2}^{\prime}\left[\frac{1}{\omega_{l 2} \pm \omega_{3} \mp \omega_{1}}+\frac{1}{\omega_{l 2} \pm \omega_{2} \mp \omega_{1}}+\frac{1}{\omega_{l 2} \pm \omega_{3} \mp \omega_{2}}\right] \text {; } \\
& p 2_{ \pm} \equiv \frac{1}{2 \hbar} \sum_{l \neq 1,2} \alpha_{12}^{\prime} \mu_{12}\left[\frac{1}{\omega_{12} \mp \omega_{3}}+\frac{1}{\omega_{l 2} \mp \omega_{2}}+\frac{1}{\omega_{l 2} \pm \omega_{1}}\right] \text {; } \\
& p 3_{i} \equiv \frac{1}{4 \hbar} \sum_{l \neq 1,2} \alpha_{2 l}^{\prime} \alpha_{l 2}^{\prime}\left[\frac{1}{\omega_{12}+2 \omega_{i}}+\frac{4}{\omega_{l 2}}+\frac{1}{\omega_{l 2}-2 \omega_{i}}\right] \text {; } \\
& p 5 \equiv \frac{1}{4 \hbar} \sum_{l \neq 1,2} \mu_{2 l} \mu_{l 1}\left[\frac{1}{\omega_{l 1}-\omega_{1}}+\frac{1}{\omega_{l 1}+\omega_{2}}\right] \text {; } \\
& p 4_{i j} \equiv \frac{1}{\hbar} \sum_{l \neq 1,2} \alpha_{2 l}^{\prime} \alpha_{l 2}^{\prime}\left[\frac{1}{\omega_{l 2}+\omega_{i}+\omega_{j}}+\frac{1}{\omega_{l 2}-\omega_{i}+\omega_{j}}\right. \\
& \left.+\frac{1}{\omega_{12}+\omega_{i}-\omega_{j}}+\frac{1}{\omega_{12}-\omega_{i}-\omega_{j}}+\frac{2}{\omega_{12}}\right] \text {; } \\
& p 6_{i j} \equiv \frac{1}{2 \hbar} \sum_{l \neq 1,2} \mu_{2 l} \alpha_{l 1}^{\prime}\left[\frac{1}{\omega_{l 1}-\omega_{i}-\omega_{j}}+\frac{1}{\omega_{l 1}-\omega_{i}+\omega_{j}}+\frac{1}{\omega_{l 1}}\right. \\
& \left.+\frac{1}{\omega_{l 1}-\omega_{i}}+\frac{1}{\omega_{l 1}+\omega_{j}}+\frac{1}{\omega_{l 1}-\omega_{j}}\right]
\end{aligned}
$$




$$
\begin{gathered}
p 7 \equiv \frac{1}{4 \hbar} \sum_{l \neq 1,2} \mu_{2 l} \alpha_{l 1}^{\prime}\left[\frac{1}{\omega_{l 1}-2 \omega_{3}}+\frac{2}{\omega_{l 1}}\right]+\frac{1}{4 \hbar} \sum_{l \neq 1,2} \mu_{l 1} \alpha_{2 l}^{\prime}\left[\frac{1}{\omega_{l 1}+\omega_{3}}+\frac{2}{\omega_{l 1}-\omega_{3}}\right] \\
p 8_{i j, \pm} \equiv \frac{1}{2 \hbar} \sum_{l \neq 1,2} \alpha_{2 l}^{\prime} \alpha_{l 1}^{\prime}\left[\frac{1}{\omega_{l 1} \mp 2 \omega_{i}}+\frac{2}{\omega_{l 1}}+\frac{2}{\omega_{l 1}+\omega_{2}-\omega_{1}}+\frac{1}{\omega_{l 1} \pm \omega_{i} \pm \omega_{j}}\right] \\
p 9 \equiv \frac{1}{\hbar} \sum_{l \neq 1,2} \alpha_{2 l}^{\prime} \alpha_{l 1}^{\prime}\left[\frac{1}{\omega_{l 1}-\omega_{1}-\omega_{3}}+\frac{1}{\omega_{l 1}-\omega_{1}+\omega_{3}}+\frac{1}{\omega_{l 1}-\omega_{1}+\omega_{2}}\right. \\
\left.+\frac{1}{\omega_{l 1}+\omega_{2}+\omega_{3}}+\frac{1}{\omega_{l 1}+\omega_{2}-\omega_{3}}+\frac{1}{\omega_{l 1}}\right] ; \\
p 10 \equiv \frac{1}{2 \hbar} \sum_{l \neq 1,2} \alpha_{2 l}^{\prime} \alpha_{l 1}^{\prime}\left[\frac{2}{\omega_{l 1}+\omega_{1}-\omega_{3}}+\frac{1}{\omega_{l 1}+\omega_{1}-\omega_{2}}+\frac{2}{\omega_{l 1}-\omega_{2}-\omega_{3}}+\frac{1}{\omega_{l 1}-2 \omega_{3}}\right] \\
i, j=1,2,3 .
\end{gathered}
$$

We define the polarization induced in this process as

$$
P=\sum_{l \neq 1,2}\left(a_{1}^{*} \mu_{11} a_{l} \mathrm{e}^{i \omega_{11} t}+a_{2}^{*} \mu_{2 l} a_{l} \mathrm{e}^{i \omega_{2 l} t}\right)+a_{1}^{*} \mu_{12} a_{2} \mathrm{e}^{i \omega_{12} t}+a_{2}^{*} \mu_{21} a_{1} \mathrm{e}^{i \omega_{21} t}+\text { c.c. },
$$

After substituting (2) into (4) we get

$$
\begin{aligned}
P & =\sum_{m}\left\{\left(\Delta r^{(m)} \cdot n+\kappa^{(m)}\right) \varepsilon_{m} \cos \Phi_{m}+\left(p c c n^{(m, n)} \cdot n+p c c^{(m, n)}\right)\right. \\
& \left.\cdot \varepsilon_{m} \varepsilon_{n} \cos \Phi_{m} \cos \Phi_{n}+\left(p s s n^{(m, n)} \cdot n+p s s^{(m, n)}\right) \cdot \varepsilon_{m} \varepsilon_{n} \sin \Phi_{m} \sin \Phi_{n}\right\} \\
& +2 s^{(-1)}\left(P_{1} \cos \Phi_{2}-P_{2} \sin \Phi_{2}\right) \cdot \varepsilon_{1}+2 s^{(-3)}\left(P_{1} \cos \theta+P_{2} \sin \theta\right) \cdot \varepsilon_{3} \\
& +2 s^{(+2)}\left(P_{1} \cos \Phi_{1}+P_{2} \sin \Phi_{1}\right) \cdot \varepsilon_{2}+4\left(P_{1} g_{1}-P_{2} g_{2}\right) \cdot \varepsilon_{1} \varepsilon_{2} \\
& +4 \mu\left\{P_{1}\left(\cos \Phi_{3} \cos \theta-\sin \Phi_{3} \sin \theta\right)+P_{2}\left(\sin \Phi_{3} \cos \theta+\cos \Phi_{3} \sin \theta\right)\right\},
\end{aligned}
$$

where $\Delta r^{(m)} \equiv \frac{1}{2}\left(r_{22}^{(m)}-r_{11}^{(m)}\right), \quad \kappa^{(m)} \equiv \frac{1}{2}\left(r_{11}^{(m)}+r_{22}^{(m)}\right), \quad r_{i i}^{(m)} \equiv \frac{2}{\hbar} \sum_{l \neq 1,2} \frac{\mu_{i l}^{2} \omega_{l i}}{\omega_{l i}^{2}-\omega_{m}^{2}}$;

$$
\begin{aligned}
& p c c n^{(m, n)} \equiv-p_{11}^{(m, n)}-p_{11}^{(-m, n)}+p_{22}^{(m, n)}+p_{22}^{(-m, n)}, \\
& p c c^{(m, n)} \equiv p_{11}^{(m, n)}+p_{11}^{(-m, n)}+p_{22}^{(m, n)}+p_{22}^{(-m, n)}, \\
& p s s n^{(m, n)} \equiv p_{11}^{(m, n)}-p_{11}^{(-m, n)}-p_{22}^{(m, n)}+p_{22}^{(-m, n)} \text {, } \\
& p s s^{(m, n)} \equiv-p_{11}^{(m, n)}+p_{11}^{(-m, n)}-p_{22}^{(m, n)}+p_{22}^{(-m, n)} \text {, } \\
& p_{i i}^{( \pm m, n)} \equiv \frac{1}{\hbar} \sum_{l \neq 1,2} \mu_{i l} \alpha_{l i}^{\prime}\left[\frac{1}{\omega_{l i} \pm \omega_{m}+\omega_{n}}+\frac{1}{\omega_{l i} \mp \omega_{m}-\omega_{n}}\right] \text {, } \\
& s^{(\mp m)} \equiv \frac{1}{2 \hbar} \sum_{l \neq 1,2} \mu_{2 l} \mu_{l 1}\left[\frac{1}{\omega_{11} \mp \omega_{m}}+\frac{1}{\omega_{12} \pm \omega_{m}}\right], \\
& P_{1} \equiv \operatorname{Re}\left(a_{1}^{*} a_{2} \mathrm{e}^{i \Delta}\right), \quad P_{2} \equiv \operatorname{Im}\left(a_{1}^{*} a_{2} \mathrm{e}^{i \Delta}\right), \quad \Delta \equiv \Delta \omega t+\left(k_{2}-k_{1}\right) z+\left(\varphi_{1}-\varphi_{2}\right), \\
& g_{1} \equiv \operatorname{Re}\{g\}, \quad g_{2} \equiv \operatorname{Im}\{g\}, \quad g \equiv \frac{1}{2 \hbar} \sum_{l \neq 1,2}\left[\frac{\mu_{11} \alpha_{l 2}^{\prime}}{\omega_{12}-\omega_{2}+\omega_{1}}+\frac{\mu_{2 l}^{*} \alpha_{l 1}^{1 *}}{\omega_{11}+\omega_{2}-\omega_{1}}\right] \text {, } \\
& \theta \equiv\left(k_{2}-k_{1}+k_{3}\right) z+\varphi_{1}-\varphi_{2}-\varphi_{3}, \quad n=\left|a_{2}\right|^{2}-\left|a_{1}\right|^{2} .
\end{aligned}
$$

The system of Equations (3) can be transformed to the equations for the pola- 
rization $P_{1,2}$ and $n$ (population difference):

$$
\begin{aligned}
\frac{\partial P_{1}}{\partial t}= & \frac{1}{\hbar}(B-F) P_{1}+\frac{1}{\hbar}\left(A-E-\Delta \omega-\frac{\partial\left(\varphi_{1}-\varphi_{2}\right)}{\partial t}\right) P_{2} \\
& +\frac{1}{2 \hbar}(D-H)+\frac{1}{2 \hbar}(D+H) n, \\
\frac{\partial P_{2}}{\partial t}= & \frac{1}{\hbar}\left(E-A-\Delta \omega+\frac{\partial\left(\varphi_{1}-\varphi_{2}\right)}{\partial t}\right) P_{1}+\frac{1}{\hbar}(B-F) P_{2} \\
& +\frac{1}{2 \hbar}(G-C)-\frac{1}{2 \hbar}(C+G) n, \\
\frac{\partial n}{\partial t}=- & \frac{2}{\hbar}(H+D) P_{1}+\frac{2}{\hbar}(C+G) P_{2}-\frac{1}{\hbar}(F+B)+\frac{1}{\hbar}(B-F) n,
\end{aligned}
$$

To make the system (6)-(8) complete we add the equations for "slowly-varying amplitudes" $\varepsilon_{1,2,3}$ and $\varphi_{1,2,3}$

$$
\begin{gathered}
\frac{\partial \varepsilon_{1}}{\partial z}+\frac{n_{1}}{c} \frac{\partial \varepsilon_{1}}{\partial t}=-\frac{2 \pi \omega_{1} N}{c n_{1}} 2 s^{(+2)} P_{2} \varepsilon_{2}, \\
\left(\frac{\partial \varphi_{1}}{\partial z}+\frac{n_{1}}{c} \frac{\partial \varphi_{1}}{\partial t}\right) \varepsilon_{1}=-\frac{2 \pi \omega_{1} N}{c n_{1}}\left(2 s^{(+2)} P_{1} \varepsilon_{2}+\left(\Delta r^{(1)} \cdot n+\kappa^{(1)}\right) \varepsilon_{1}\right), \\
\frac{\partial \varepsilon_{2}}{\partial z}+\frac{n_{2}}{c} \frac{\partial \varepsilon_{2}}{\partial t}=-\frac{2 \pi \omega_{2} N}{c n_{2}}\left(-2 s^{(-1)}\right) P_{2} \varepsilon_{1}, \\
\left(\frac{\partial \varphi_{2}}{\partial z}+\frac{n_{2}}{c} \frac{\partial \varphi_{2}}{\partial t}\right) \varepsilon_{2}=-\frac{2 \pi \omega_{2} N}{c n_{2}}\left(2 s^{(-1)} P_{1} \varepsilon_{1}+\left(\Delta r^{(2)} \cdot n+\kappa^{(2)}\right) \varepsilon_{2}\right), \\
\frac{\partial \varepsilon_{3}}{\partial z}+\frac{n_{3}}{c} \frac{\partial \varepsilon_{3}}{\partial t}=-\frac{2 \pi \omega_{3} N}{c n_{3}} 4 \mu\left(P_{2} \cos \theta-P_{1} \sin \theta\right), \\
\left(\frac{\partial \varphi_{3}}{\partial z}+\frac{n_{3}}{c} \frac{\partial \varphi_{3}}{\partial t}\right) \varepsilon_{3}=-\frac{2 \pi \omega_{3} N}{c n_{3}} 4 \mu\left(P_{1} \cos \theta+P_{2} \sin \theta\right),
\end{gathered}
$$

where

$$
\begin{aligned}
A \equiv & r_{1} \cdot \varepsilon_{1}^{2}+r_{2} \cdot \varepsilon_{2}^{2}+r_{3} \cdot \varepsilon_{3}^{2}+\left[r 1_{+}+r 1_{-}+r 2_{+}+r 2_{-}\right] \cdot \varepsilon_{1} \varepsilon_{2} \varepsilon_{3} \cos \theta \\
& +r 3_{1} \cdot \varepsilon_{1}^{4}+r 3_{2} \cdot \varepsilon_{2}^{4}+r 3_{3} \cdot \varepsilon_{3}^{4}+r 4_{12} \cdot \varepsilon_{1}^{2} \varepsilon_{2}^{2}+r 4_{13} \cdot \varepsilon_{1}^{2} \varepsilon_{3}^{2}+r 4_{23} \cdot \varepsilon_{2}^{2} \varepsilon_{3}^{2}
\end{aligned}
$$

$$
B \equiv\left[r 1_{+}-r 1_{-}-r 2_{+}+r 2_{-}\right] \cdot \varepsilon_{1} \varepsilon_{2} \varepsilon_{3} \sin \theta \text {, }
$$

$$
\begin{gathered}
C \equiv \frac{1}{2} \mu_{12} \cdot \varepsilon_{3} \cos \theta+\left[\alpha_{12}^{\prime}+r 5\right] \varepsilon_{1} \varepsilon_{2}+\left[r 6_{31} \cdot \varepsilon_{1}^{2} \varepsilon_{3}+r 6_{32} \cdot \varepsilon_{2}^{2} \varepsilon_{3}+r 7 \cdot \varepsilon_{3}^{3}\right] \cos \theta \\
+r 8_{12,+} \varepsilon_{1}^{3} \varepsilon_{2}+r 8_{21,-} \varepsilon_{1} \varepsilon_{2}^{3}+r 9 \cdot \varepsilon_{1} \varepsilon_{2} \varepsilon_{3}^{2}+r 10 \cdot \varepsilon_{1} \varepsilon_{2} \varepsilon_{3}^{2} \cos 2 \theta \\
D \equiv \frac{1}{2} \mu_{12} \cdot \varepsilon_{3} \cdot \sin \theta+\left[r 6_{31} \cdot \varepsilon_{1}^{2} \varepsilon_{2}+r 6_{32} \cdot \varepsilon_{2}^{2} \varepsilon_{3}+r 7 \cdot \varepsilon_{3}^{3}\right] \cdot \sin \theta \\
\quad+r 10 \cdot \varepsilon_{1} \varepsilon_{2} \varepsilon_{3}^{2} \sin 2 \theta \\
E \equiv p_{1} \cdot \varepsilon_{1}^{2}+p_{2} \cdot \varepsilon_{2}^{2}+p_{3} \cdot \varepsilon_{3}^{2}+\left[p 1_{+}+p 1_{-}+p 2_{+}+p 2_{-}\right] \cdot \varepsilon_{1} \varepsilon_{2} \varepsilon_{3} \cos \theta \\
+p 3_{1} \cdot \varepsilon_{1}^{4}+p 3_{2} \cdot \varepsilon_{2}^{4}+p 3_{3} \cdot \varepsilon_{3}^{4}+p 4_{12} \cdot \varepsilon_{1}^{2} \varepsilon_{2}^{2}+p 4_{13} \cdot \varepsilon_{1}^{2} \varepsilon_{3}^{2}+p 4_{23} \cdot \varepsilon_{2}^{2} \varepsilon_{3}^{2} \\
F \equiv\left[p 1_{+}-p 1_{-}-p 2_{+}+p 2_{-}\right] \cdot \varepsilon_{1} \varepsilon_{2} \varepsilon_{3} \sin \theta
\end{gathered}
$$

$$
\begin{aligned}
G \equiv & \frac{1}{2} \mu_{21} \cdot \varepsilon_{3} \cos \theta+\left[\alpha_{21}^{\prime}+p 5\right] \cdot \varepsilon_{1} \varepsilon_{2}+\left[p 6_{32} \cdot \varepsilon_{2}^{2} \varepsilon_{3}+p 6_{32} \cdot \varepsilon_{2}^{2} \varepsilon_{3}+p 7 \cdot \varepsilon_{3}^{3}\right] \cos \theta \\
& +p 8_{12,+} \cdot \varepsilon_{1}^{3} \varepsilon_{2}+p 8_{21,-} \cdot \varepsilon_{1} \varepsilon_{2}^{3}+p 9 \cdot \varepsilon_{1} \varepsilon_{2} \varepsilon_{3}^{2}+p 10 \cdot \varepsilon_{1} \varepsilon_{2} \varepsilon_{3}^{2} \cos 2 \theta
\end{aligned}
$$




$$
\begin{aligned}
H \equiv & \frac{1}{2} \mu_{21} \cdot \varepsilon_{3} \sin \theta+\left[p 6_{31} \cdot \varepsilon_{1}^{2} \varepsilon_{2}+p 6_{32} \cdot \varepsilon_{2}^{2} \varepsilon_{3}+p 7 \cdot \varepsilon_{3}^{3}\right] \sin \theta \\
& +p 10 \cdot \varepsilon_{1} \varepsilon_{2} \varepsilon_{3}^{2} \sin 2 \theta
\end{aligned}
$$

\section{Gain Factor G}

Such significant characteristics of crystals as the dispersion relation and damping of phonon-polaritons have been the subject of many both theoretical and experimental investigations in recent years. For instance, the experimental methods used in these studies include the impulsive SRS and transient grating experiments with femtosecond pulses [16] [17], and coherent anti-Stokes Raman Scattering (CARS) with picosecond pulses [18]. The information on the damping of polaritons is contained in Raman linewidth, which can be obtained from the stimulated gain curve [19]. This method was successfully applied in [20], where the two-beam amplifier experiment SRS gain measurements were performed in $\mathrm{LiNbO}_{3}$ for the polariton frequencies ranging from 30 to $230 \mathrm{~cm}^{-1}$. The measurements were carried out at liquid-nitrogen temperature in doped congruent $\mathrm{LiNbO}_{3}$ and undoped nearly stoichiometric $\mathrm{LiNbO}_{3}$. It was the comparison of doped and nearly stoichiometric crystals which provided new information on the number and type of low-frequency excitations determining the polariton damping over almost complete frequency range.

Now we show that our equations are consistent with the experimental results presented in [20]. The theoretical treatment of the gain factor for SRS by polaritons is based on the solutions of the coupled wave equations for the Stokes and polariton fields. In quasi-stationary case, in absence of phase modulation of any kind, and given pump field, the system of equations for the $\varepsilon_{2}$ (Stokes) and $\varepsilon_{3}$ (polariton) can be reduced to the following (see (11) and (13)):

$$
\begin{gathered}
\frac{\partial \varepsilon_{2}}{\partial z}=i \frac{1}{2 k_{s}}\left(k_{s}^{2}-\frac{\omega_{s}^{2}}{c^{2}} \tilde{\varepsilon}_{s}\right) \varepsilon_{2}-i \frac{\omega_{s}^{2}}{2 k_{s} c^{2}} \chi_{s} \varepsilon_{1} \varepsilon_{3}^{*} \\
\frac{\partial \varepsilon_{3}}{\partial z}=i \frac{1}{2 k_{p}}\left(k_{p}^{2}-\frac{\omega_{p}^{2}}{c^{2}} \tilde{\varepsilon}_{p}\right) \varepsilon_{3}-i \frac{1}{2 k_{p}} \frac{\omega_{p}^{2}}{c^{2}} \chi_{p} \varepsilon_{1} \varepsilon_{2}^{*}
\end{gathered}
$$

where $k_{s, p}=\frac{\omega_{s, p}}{c} n_{s, p}, \quad \tilde{\varepsilon}_{s, p}$ are the dielectric constants, $\chi_{s, p}$ are the nonlinear coefficients. This system is the part of more complex system considered in [21]. When we assume that there is exponential amplification of the electromagnetic field with a gain factor $G$ and after the insertion of the electromagnetic fields expressed as $A_{s, p} \mathrm{p}^{\frac{1}{2} G_{s} z}$ into (15) and (16) we get the expression for $G$ as

$$
G \approx \frac{v_{s}}{n_{s}} \frac{1}{\tilde{\varepsilon}_{p}^{\prime \prime}} \chi^{\prime \prime 2} \varepsilon_{1}^{2},
$$

which is completely consistent with the expression of the gain given in [20] and [22]. Therefore, the gain factor is related to the dielectric function of the crystal, which contains the damping of the polariton. 
Here $v_{s}=\frac{\omega_{s}}{2 \pi c} ; n_{s}$ is the index of refraction at Stokes frequency;

$\varepsilon_{p}^{\prime \prime}=\sum_{f} s_{f} \frac{v_{f}^{2} \gamma_{f} v_{p}}{\left(v_{f}^{2}-v_{p}^{2}\right)^{2}+\gamma_{f}^{2} v_{p}^{2}} ; s_{f}$ is the oscillator's strength of the dipole-ac-

tive phonon $v_{f} ; \gamma_{f}$ is the damping constant; $\chi^{\prime \prime}$ is the imaginary part of quadratic polarizability. The expression for $\chi^{\prime \prime}$ is given in [23]:

$$
\chi^{\prime \prime}=\sum_{f} \chi_{f} \frac{v_{f}^{2} \gamma_{f} v_{p}}{\left(v_{f}^{2}-v_{p}^{2}\right)^{2}+\gamma_{f}^{2} v_{p}^{2}}
$$

where $\chi_{f}=\frac{1}{8 \pi^{3}} \frac{1}{\sqrt{\hbar c}} \frac{1}{v_{l}^{2}}\left(\frac{s_{f} \sigma_{f}}{v_{f}}\right)^{\frac{1}{2}}, . \sigma_{f}$ is the effective cross-section of combinational scattering.

In calculations we used for $G$ in $\mathrm{LiNbO}_{3}$ the following (see [20]): $I_{l} \approx 10 \mathrm{MW} / \mathrm{cm}^{2} ; \chi_{f} \approx 10^{-8} \mathrm{esu} ; \mathrm{ZnO}$-doped $\mathrm{LiNbO}_{3}: v_{f}\left(\mathrm{~cm}^{-1}\right)=112,122$, $124,150,163,190,199,222$, and 235; $\gamma_{f}\left(\mathrm{~cm}^{-1}\right)=33,20,12,12,14,12,11,14$, and 14; nearly stoichiometric $\mathrm{LiNbO}_{3}: v_{f}\left(\mathrm{~cm}^{-1}\right)=106,125,148,153,167,188$, $198,217,233$, and 239; $\gamma_{f}\left(\mathrm{~cm}^{-1}\right)=33,20,10,8,20,13,3,10,10$, and 4 . The gain factor $\mathrm{G}$ as a function of the polariton frequency in nearly stoichiometric $\mathrm{LiNbO}_{3}$ is shown in Figure 1. The gain factor $\mathrm{G}$ versus polariton frequency in $\mathrm{ZnO}: \mathrm{LiNbO}_{3}$ is shown in Figure 2. In both graphs the squares represent experimental points ([20]), whereas the solid curves are the simulation based on (17).

\section{Analysis of Basic System}

In this section we provide the analysis of the system (6)-(14) assuming that the all amplitudes of electromagnetic waves are real $\left(\varphi_{1,2,3}=0\right)$ and the waves are synchronous $(\theta \approx 0)$. Only low-order nonlinear processes are considered. The simplified system can be written as follows:

$$
\begin{gathered}
\frac{\partial n}{\partial t}=\frac{2}{\hbar}\left(\mu \varepsilon_{3}+2 \alpha^{\prime} \varepsilon_{1} \varepsilon_{2}\right) P_{2}, \\
\frac{\partial P_{2}}{\partial t}=-\frac{1}{2 \hbar}\left(\mu \varepsilon_{3}+2 \alpha^{\prime} \varepsilon_{1} \varepsilon_{2}\right) n, \\
\frac{\partial \varepsilon_{1}}{\partial z}+\frac{n_{1}}{c} \frac{\partial \varepsilon_{1}}{\partial t}=-\frac{2 \pi \omega_{1} N}{c n_{1}} 2 s^{(+2)} P_{2} \varepsilon_{2}, \\
\frac{\partial \varepsilon_{2}}{\partial z}+\frac{n_{2}}{c} \frac{\partial \varepsilon_{2}}{\partial t}=-\frac{2 \pi \omega_{2} N}{c n_{2}}\left(-2 s^{(-1)}\right) P_{2} \varepsilon_{1}, \\
\frac{\partial \varepsilon_{3}}{\partial z}+\frac{n_{3}}{c} \frac{\partial \varepsilon_{3}}{\partial t}=-\frac{2 \pi \omega_{3} N}{c n_{3}} 4 \mu P_{2},
\end{gathered}
$$

where $n_{1,2,3}$ are linear indices of refraction $n_{1,2,3} \approx \sqrt{\tilde{\varepsilon}_{1,2,3}}$.

The solutions for $n$ and $P_{2}$ can be expressed as

$$
n=-\cos \psi, P_{2}=\frac{1}{2} \sin \psi,
$$




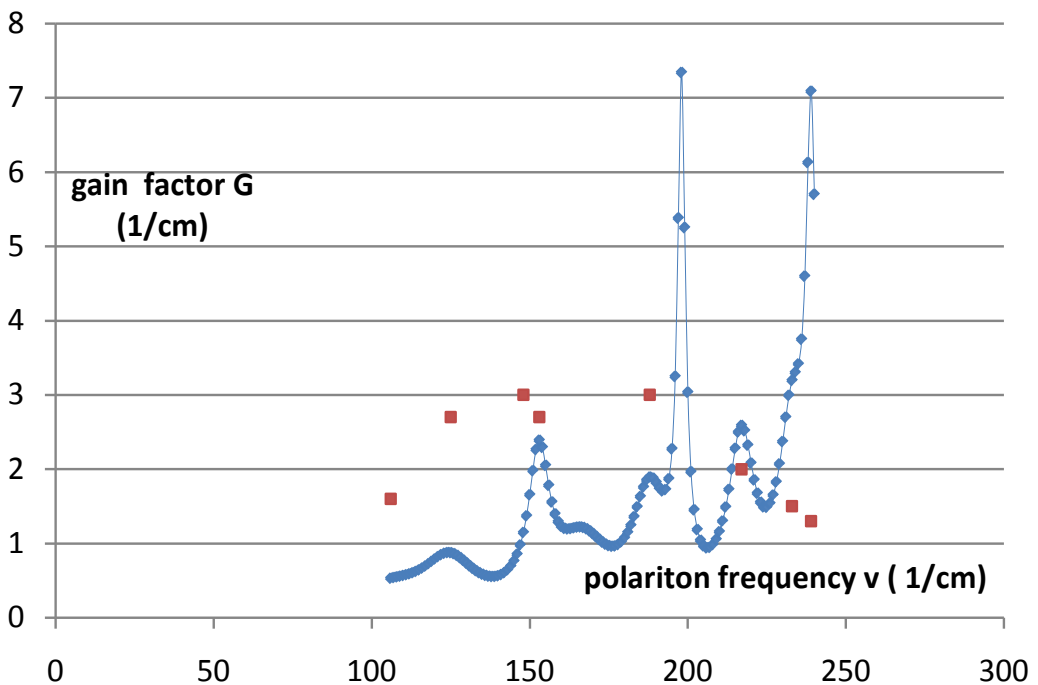

Figure 1. Gain factor $G$ versus polariton frequency in nearly stoichiometric $\mathrm{LiNbO}_{3}$. Squares: experimental points ([20]); solid curve: simulation based on (17).

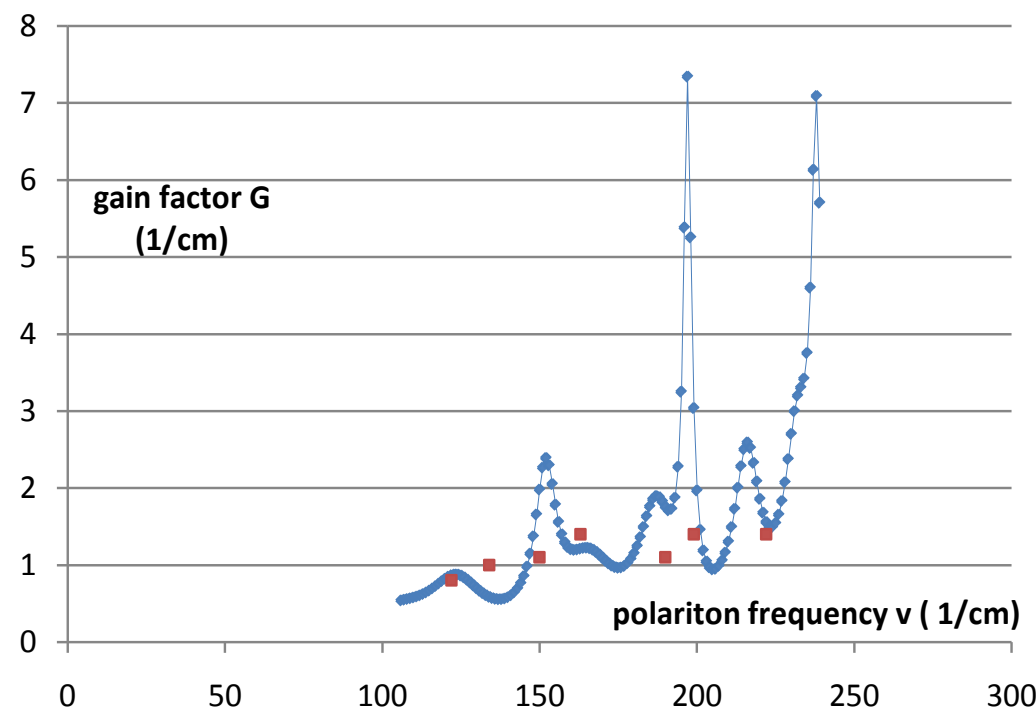

Figure 2. Gain factor $\mathrm{G}$ versus polariton frequency in $\mathrm{ZnO}$-doped $\mathrm{LiNbO}_{3}$. Squares: experimental points ([20]); solid curve: simulation based on (17).

where $\psi=\frac{1}{\hbar} \int_{-\infty}^{t}\left(\mu \varepsilon_{3}+2 \alpha^{\prime} \varepsilon_{1} \varepsilon_{2}\right) \mathrm{d} t$

1) The system of Equations (19)-(23) includes both the classical case of resonant interaction of electromagnetic wave with two-level system (See [26]) $\left(\alpha^{\prime}=0\right)$ and the one that corresponds to the combinational scattering by nonpolar optical phonons studied in [27] $(\mu=0)$.

2) The extreme case $\alpha^{\prime}=0$ also results in standard "area theorem" for $\varepsilon_{3}$ (See [26]):

$$
\frac{\mathrm{d} W_{3}}{\mathrm{~d} z}=-\frac{8 \pi \hbar \omega_{3} N}{c n_{3}}(1-\cos \Phi),
$$


where $\Phi=\frac{\mu}{\hbar} \int_{-\infty}^{+\infty} \varepsilon_{3}(z, t) \mathrm{d} t, W_{3} \equiv \int_{-\infty}^{+\infty} \varepsilon_{3}^{2}(z, t) \mathrm{d} t$.

3) In the absence of dispersion $\left(n_{1} \approx n_{2} \approx n_{3} \approx \bar{n}\right)$ the system (19)-(23) can be reduced to Sine-Gordon equation

$$
\frac{\partial^{2} \psi}{\partial \tau \partial \varsigma}=-\sin \psi
$$

where $\tau=c a\left(t-\frac{z \bar{n}}{c}\right), \quad \varsigma=a z, \quad a^{2}=\frac{4 \pi N}{\hbar c^{2} \bar{n}}\left[\mu^{2} \omega_{3}+\alpha^{\prime} C\right]$,

$\omega_{1} s^{(+2)} \varepsilon_{2}^{2}-\omega_{2} s^{(-1)} \varepsilon_{1}^{2} \equiv C \quad$ (the given field approximation for $\varepsilon_{1,2}$ ).

4) If we introduce a new variable $\tau=t-\frac{z}{v}$ ( $v$ is the speed of the pulse), we can reduce the system (19)-(23) to the equation of the motion of physical pendulum from the position of upper unstable equilibrium:

$$
\frac{\partial^{2} \psi}{\partial \tau^{2}}=\frac{1}{\tau_{p}^{2}} \sin \psi
$$

where $\kappa_{1,2,3}^{-1}=\frac{n_{1,2,3}}{c}-\frac{1}{v}, \quad \tau_{p}^{-2}=\frac{4 \pi N}{\hbar c}\left[-\frac{\omega_{3} \kappa_{3} \mu^{2}}{n_{3}}+\alpha^{\prime} \tilde{C}\right]$,

$$
\tilde{C} \equiv-\frac{\omega_{1} \kappa_{1} s^{(+2)}}{n_{1}} \varepsilon_{2}^{2}+\frac{\omega_{2} \kappa_{2} s^{(-1)}}{n_{2}} \varepsilon_{1}^{2} \quad \text { (the given field approximation for } \varepsilon_{1,2} \text { ). }
$$

5) We also provided the numerical solution of (19)-(23). To do that, we brought that system to unitless form:

$$
\begin{gathered}
\frac{\partial \tilde{\varepsilon}_{1}}{\partial \tilde{z}}+n_{1} \frac{\partial \tilde{\varepsilon}_{1}}{\partial \tilde{t}}=-C 1 \cdot P_{2} \tilde{\varepsilon}_{2}, \\
\frac{\partial \tilde{\varepsilon}_{2}}{\partial \tilde{z}}+n_{2} \frac{\partial \tilde{\varepsilon}_{2}}{\partial \tilde{t}}=C 2 \cdot P_{2} \tilde{\varepsilon}_{1}, \\
\frac{\partial \tilde{\varepsilon}_{3}}{\partial \tilde{z}}+n_{3} \frac{\partial \tilde{\varepsilon}_{3}}{\partial \tilde{t}}=-C 3 \cdot P_{2}, \\
\frac{\partial P_{2}}{\partial \tilde{t}}=-\left(C 4 \cdot \tilde{\varepsilon}_{3}+C 5 \cdot \tilde{\varepsilon}_{1} \tilde{\varepsilon}_{2}\right) n, \\
\frac{\partial n}{\partial t}=\left(C 4 \cdot \tilde{\varepsilon}_{3}+C 5 \cdot \tilde{\varepsilon}_{1} \tilde{\varepsilon}_{2}\right) P_{2},
\end{gathered}
$$

where $\tilde{\varepsilon}_{1,2,3}=\varepsilon_{1,2,3} / A_{0}, \tilde{t}=t / \tau_{0}, \tilde{z}=z / z_{0}, z_{0}=c \tau_{0}, C 1 \equiv \frac{2 \pi \omega_{1} N z_{0}\left(2 s^{(+2)}\right)}{c n_{1}}$, $C 2 \equiv \frac{2 \pi \omega_{2} N z_{0}\left(2 s^{(-1)}\right)}{c n_{2}}, C 3 \equiv \frac{2 \pi \omega_{3} N 4 \mu z_{0}}{c n_{3} A_{0}}, C 4 \equiv \frac{\tau_{0} \mu A_{0}}{2 \hbar}, C 5 \equiv \frac{\alpha^{\prime} \tau_{0} A_{0}^{2}}{\hbar} ;$

$\tau_{0}$ and $A_{0}$ are characteristic time interval and amplitude of electromagnetic wave; $N$ is the number of atoms in $\mathrm{cm}^{3} ; \mu$ is the average dipole moment.

The space-time evolution of the polariton wave at frequency $\omega_{3}$ is shown in Figure $3\left(\mathrm{I} 31=\tilde{\varepsilon}_{3}^{2}(\tilde{z}, \tilde{t}) / \tilde{\varepsilon}_{1, \max }^{2} ; C 1=C 2=C 4=C 5=1 ; C 3=15 ; z^{*}=z / z_{0} ;\right.$ $t^{*}=t / t_{0} ; \quad N \approx 10^{19} \mathrm{~cm}^{-3}, \quad \tau_{0} \approx 10^{-10} \mathrm{~s}, \quad \mu \approx 10^{-18}$ esu $)$. 


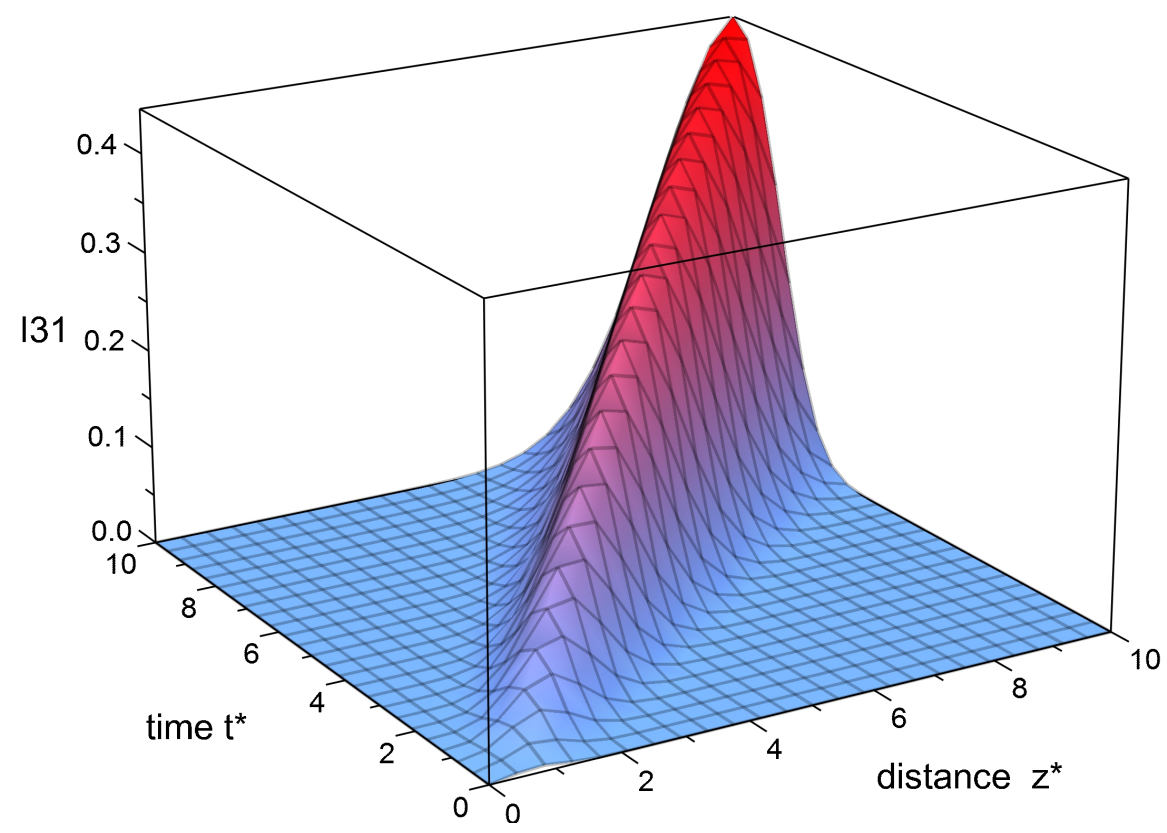

Figure 3. The space-time evolution of the normalized polariton intensity I31.

\section{Conclusions}

In this paper we have found the following:

1) The system of Equations (6)-(14) that models the processes of nonstationary SRS by polaritons in nonlinear media consisting of polar optical phonons is obtained;

2) It has been shown that the frequency dependence of the gain factor of the Stokes matches with the experimental results;

3) The simplified system of Equations (19)-(23) (for real amplitudes of all electromagnetic waves and "low-order" nonlinear processes);

4) It is shown that the latter system could be reduced to either case of purely combinational interaction (the nonstationary SRS by nonpolar phonons $(\mu=0)$ ) or the classical case of nonlinear resonant (but not combinational $\left.\left(\alpha^{\prime}=0\right)\right)$ interaction (including "area theorem") between the electromagnetic field and system;

5) It is also shown that (19)-(23) could be reduced to the standard Sine-Gordon equation;

6) The conditions at which the system (19)-(23) becomes the equation of the motion of physical pendulum;

7) The numerical analysis of (19)-(23) has indicated the possibility of effective conversion to infrared radiation, which could be useful for the design of wideband frequency converters.

\section{References}

[1] Angelakis, D.G. (2017) Quantum Simulations with Photons and Polaritons. Springer, Berlin. https://doi.org/10.1007/978-3-319-52025-4

[2] Logoudakis, K. (2013) The Physics of Exciton-Polariton Condensates. CRC Press, 
Boca Raton. https://doi.org/10.1201/b15531

[3] Kavokin, A. and Malpuech, G. (2003) Cavity Polaritons. Elsevier, New York.

[4] Sanvitto, D. and Timofeev, V. (2012) Exciton Polaritons in Microcavities: New Frontiers. Springer, Heidelberg.

[5] Schubert, M. (2004) Infrared Ellipsometry of Semiconductor Layer Structures. Springer, Leipzig.

[6] Tarkhanyan, R.H. and Uzunglu, N.K. (2006) Radiowaves and Polaritons in Anizotropic Media (Uniaxial Semiconductors). WILEY-VCH Verlag GmbH\&Co. KGaA, Weinheim. https://doi.org/10.1002/3527608125

[7] Feurer, T., Stoyanov, N.S., Ward, D.W., Vaughan, J.C., Statz, E.R. and Nelson, K.A. (2007) Terahertz Polaritonics. Annual Review of Materials Research, 27, 317-350. https://doi.org/10.1146/annurev.matsci.37.052506.084327

[8] Kojima, S., Tsumura, N., Takeda, M.W. and Nishizawa, S. (2003) Far-Infrared Phonon-Polariton Dispersion Probed by Terahertz Time-Domain Spectroscopy. Physical Review, B67, 035102-035103. https://doi.org/10.1103/PhysRevB.67.035102

[9] Claus, R., Merten, L. and Brandmuller (2006) Light Scattering by Phonons-Polaritons. Springer-Verlag, Berlin.

[10] Agranovich, V.M. and Mills, D.L. (2012) Surface Polaritons: Electromagnetic Waves at Surfaces and Interfaces. Elsevier, New York.

[11] Grimmins, T.F., Stoyanov, N.S. and Nelson, K.A. (2002) Heterodyned Impulsivestimulated Raman Scattering Of Phonon-Polaritons in $\mathrm{LiTaO}_{3}$ and $\mathrm{LiNbO}_{3}$. The Journal of Chemical Physics, 117, 2882-2887. https://doi.org/10.1063/1.1491948

[12] Kornienko, M.E. and Mikhnytsky, S.I. (2002) Medium Wave Bleaching and HighEfficient Generation of Radiation under Stimulated Raman Scattering by Polaritons. Ukrainian Journal of Physics, 47, 726-737.

[13] Pasiskevicius, V., Canalias, C. and Laurell, F. (2006) Highly Efficient Stimulated Raman Scattering of Picosecond Pulses in $\mathrm{KTiOPO}_{4}$. Applied Physics Letters, 88, 041110-041113. https://doi.org/10.1063/1.2166683

[14] Torii, K., Ono, M., Sota, T., Azunata, T., Chichibu, S.F. and Nakamura, S. (2000) Raman Scattering from Phonon-Polaritons in GaN. Physical Review B, 62, 10861. https://doi.org/10.1103/PhysRevB.62.10861

[15] Poluektov, I.A. (1977) On Third-Harmonic Generation under Double-Quantum Interaction between High-Power Light Pulses and Resonant Media. Quantum Electronics, 4, 653-657.

[16] Bakker, H.I., Hunsche, S. and Kurz, H. (1994) Investigation of Anharmonic Lattice Vibrations with Coherent Phonon Polaritons. Physical Review B, 50, 914-921. https://doi.org/10.1103/PhysRevB.50.914

[17] Wiederrecht, G.P., Dougherty, T.P., Phar, L., Nelson, K.A., Leaird, D.E. and Weiner, A.M. (1995) Explanation of Anomalous Polaritons Dynamics in $\mathrm{LiTaO}_{3}$. Physical Review B, 51, 916-932.

[18] Qiu, T. and Maier, M. (1997) Long Distance Propagation and Damping of LowFrequency Phonon Polaritons in $\mathrm{LiNbO}_{3}$. Physical Review B, 56, R5717-R5721.

[19] Grigiryan, G.G., Guzalyan, R.N., Crylow, V.N., Paperny, S.B., Strizevskii, V.L. and Feshchenko, V.P. (1983) Spontaneous and Stimulated Combinational Scattering of Light by Polaritons in $\mathrm{LiIO}_{3}$. Bulletin of the Russian Academy of Sciences. Physics, 47, 1940-1943.

[20] Schwarz, U.T. and Mayer, M. (1998) Damping Mechanisms of Phonon Polaritons, Exploited by Stimulated Raman Gain Measurements. Physical Review B, 58, 
766-775. https://doi.org/10.1103/PhysRevB.58.766

[21] Marchevskii, F.N., Strizhevskii, V.L. and Feshchenko, V.P. (1984) Soliton Generation by Stimulated Raman Scattering with Excitation of Polar Optical Phonons. Soviet Journal of Quantum Electronics, 14, 277-281. https://doi.org/10.1070/QE1984v014n02ABEH004691

[22] Barker Jr., A.S. and Loudon, R. (1972) Response Functions in the Theory of Raman Scattering by Vibrational and Polariton Models in Dielectric Crystals. Reviews of Modern Physics, 44, 18-48. https://doi.org/10.1103/RevModPhys.44.18

[23] Strizhevskii, V.L. (1972) Theory of Stimulated Raman Scattering by Polaritons in Cubic and Uniaxial Crystals. Journal of Experimental and Theoretical Physics, 62, 1446-1460.

[24] Otaguro, W., Wiener-Avnear, E., Arguello, C.A. and Porto, S.P.S. (1971) Phonons, Polaritons, and Oblique Phonons in $\mathrm{LiIO}_{3}$ by Raman Scattering and Infrared Reflection. Physical Review B, 4, 4542-4551. https://doi.org/10.1103/PhysRevB.4.4542

[25] Montgomery Jr., G.P. and Giallorenzi, T.G. (1973) Experimental and Theoretical Study of Parametric and Polar Scattering in $\mathrm{LiIO}_{3}$ and $\mathrm{LiNbO}_{3}$. Physical Review $B$, 8, 808-823. https://doi.org/10.1103/PhysRevB.8.808

[26] McCall, S.L. and Mahn, E.L. (1969) Self-Induced Transparency. Physical Review Journals Archive, 183, 457-486. https://doi.org/10.1103/PhysRev.183.457

[27] Makhviladze, T.M., Sarychev, M.E. and Shelepin, L.A. (1975) Raman Scattering of Light by an Excited Medium. Journal of Experimental and Theoretical Physics, 69, 499-512.

[28] Mandel, P. (2010) Nonlinear Optics. Wiley, Toronto.

[29] Sakurai, I.I. and Napolitano, I. (2013) Modern Quantum Mechanics. Second Edition, Pearson, New-York.

[30] Davydov, A.S. and Haar, D. (2016) Quantum Mechanics. Second Edition, Science Direct, Amsterdam. 